\title{
BINARY SHIFTS OF HIGHER COMMUTANT INDEX
}

\author{
GEOFFrey L. PRICE
}

Abstract. In a previous paper the author has shown that all binary shifts of commutant index 2 are cocycle conjugate. In this paper we show that there are only finitely many conjugacy classes of binary shifts of commutant index 3 .

Mathematics subject classification (2010): 46L10,46L40,46L55. matrix.

Keywords and phrases: binary shift; cocycle conjugacy; commutant index; center sequence; Toeplitz

\section{REFERENCES}

[1] W. Arveson And G. Price, The structure of spin systems, Internat. J. Math. 14 (2003), 119-137.

[2] D. Bures AND H. Yin, Outer conjugacy of shifts on the hyperfinite $I_{1}$ factor, Pacific J. Math. 142 (1990), 245-257.

[3] A. ConNES, Periodic automorphisms of the hyperfinite factor of type $I I_{1}$, Acta Sci. Math. 17 (1977), $39-66$.

[4] E. Enomoto and Y. Watatani, Powers' binary shifts on the hyperfinite factor of type $I_{1}$, Proc. Amer. Math. Soc. 105 (1989), 371-374.

[5] V. F. R. JONES, Index for subfactors, Invent. Math. 72 (1983), 1-25.

[6] R. LidL AND H. NiederReITER, Introduction to finite fields and their applications, Cambridge Univ. Press, 1987.

[7] M. Newman, Integral matrices, Academic Press, 1986.

[8] R. T. POWERS, An index theory for semigroups of $*$-endomorphisms of $\mathscr{B}(\mathscr{H})$ and type $I I_{1}$ factors, Canad. J. Math 40 (1988), 86-114.

[9] R. T. POWERS AND G. PRICE, Cocycle conjugacy classes of shifts on the hyperfinite II factor, J. Funct. Anal. 121 (1994), 275-295.

[10] G. PRICE, Cocycle conjugacy classes of shifts on the hyperfinite $I I_{1}$ factor II, J. Operator Theory 39 (1998), 177-195.

[11] G. PRICE, Shifts on the hyperfinite II factor, J. Functional Analysis 156 (1998), 121-169.

[12] G. PRICE, Shifts on type II factors, Canad. J. Math 39 (1987), 492-511.

[13] G. PRICE AND G. H. TRUitT, On the ranks of Toeplitz matrices over finite fields, Linear Algebra and its Applications 294 (1999), 49-66. 